\title{
LIFTING THE COVID-19 LOCKDOWN: DIFFERENT SCENARIOS FOR FRANCE
}

\author{
Emmanuelle Augeraud-VÉron*,†
}

\begin{abstract}
A mathematical model has been developed to study the spread of the COVID-19 epidemic in France. To calibrate the model, data from the French Public Health Agency were examined. The spread of the epidemic greatly depends on lockdown measures (referred to in France as 'confinement'). The aim of this paper is to predict the expected evolution of the epidemic based on the various possible scenarios for ending the lockdown. The spread of the disease, and its re-emergence, will be determined by these scenarios.
\end{abstract}

Mathematics Subject Classification. 92.10, 92D30.

Received April 7, 2020. Accepted July 26, 2020.

\section{INTRODUCTION}

In December 2019, the World Health Organization warned of several cases of pneumonia in Wuhan, China. On January 7th 2020, China confirmed that a new virus, of the coronavirus family, referred to as COVID-19 was responsible for this pneumonia. On March 11th, the World Health Organization officially declared a pandemic, and by March 22nd, there were 81,500 confirmed cases in China, with 3,267 deaths. At this time, some 188 countries around the world had been affected. However, prior to this, on March 18th 2020, China had already stated that no new cases of the virus had been reported for the first time since the beginning of the epidemic. Stopping the spread of this virus in China was made possible by lockdown. In fact, by the end of January 2020, due to the extent of the spread of the virus across China, its government had set drastic lockdown measures in place.

In France, the coronavirus was identified on January 24th 2020, when the first three cases were recorded. As of February 29th, 100 individuals were already reported to have the disease, with 2 deaths being recorded. Therefore, Stage 2 of the ORSAN plan was activated with the aim of slowing down the spread of the virus. By March 14th, with some 4500 reported cases and 91 deaths, the epidemic was raised to Stage 3, with all non-essential services being closed to the general public. Since March 17th, all citizens have been confined to their homes, except for certain justifiable reasons, and movement has been limited to a strict minimum.

By March 25th, it was estimated that more than 100,000 fines had been issued for non-compliance with the lockdown measures (Mignon Colombet and Floreancig [13]). In fact, since March 17th, the means for dealing with those who contravene the lockdown measures have become more stringent, with a fine of 135 euros being

Keywords and phrases: COVID-19, confinement, public health policy.

University of Bordeaux, GREThA, 16 avenue Léon Duguit,CS 50057, 33608 Pessac Cedex, France.

* Corresponding author: emmanuelle.augeraud@u-bordeaux.fr

$\dagger$ The author was supported by the ANR flash COVID-19: MPCUII grant. 
introduced for any violations of non-justified movement. Two days later, five people who had been fined several times for non-respect of the lockdown measures were taken into police custody for endangering the lives of others. The government has had to take more coercive measures in the face of non-compliance with these lockdown measures, and the Courts have had to impose a prison sentence on the basis of endangerment of life (Article 223-1 of French Penal Code).

Lockdown ended in France on May 11th, with many restrictions (school closures, movement restrictions ....). This partial lockdown lift (called phase 1) has enable to contain the epidemics. The decree of May 31st (Decret 2020-663) organizing the entry into phase 2 of the lift of lockdown, published in the Official Journal specifies all measures concerning travel and public transport, shops, establishments and places open or closed after June 2nd 2020 until June 21st. From June 22nd started phase three, with obligation to go to school for all students.

The issue that needs to be considered is the future evolution of the virus when this lockdown is lifted. Different scenarios, depending on the application of advices for stopping the virus spread, will be considered.

\section{MODEL AND DATA}

We consider an SLIAR compartmental model with susceptible $(S)$, latent infectious $(L)$, symptomatic infectious $(I)$ and asymptomatic (or mild symptomatic) infectious $(A)$. This SLIAR model has been considered by Arino et al. [2] to describe diseases such as influenza. Susceptible individuals $S(t)$ can be contaminated by all infectious individuals and become latent infectious asymptomatic $(L(t))$ then, according to their symptoms, they can become either infectious asymptomatic (or mild symptomatic) individuals $(A(t))$ or infectious severe symptomatic $(I(t))$. We assume that infectious asymptomatic develop severe symptoms at rate $f \nu$ with $f \in] 0,1[$ , and they have mild symptoms at rate $(1-f) \nu$, where $\nu^{-1}$ is the average duration of the asymptomatic period. Asymptomatic individuals recovers at rate $\eta$, and severe symptomatic individuals may either recover at rate $\eta$ or die at rate $\gamma$. The spread of the disease across the population is considered in the following system

$$
\left\{\begin{array}{l}
S^{\prime}=-\tau S\left(\varepsilon_{1}(t)(L+A)+\varepsilon_{2} I\right) \\
L^{\prime}=\tau S\left(\varepsilon_{1}(t)(L+A)+\varepsilon_{2} I\right)-\nu L \\
I^{\prime}=f \nu L-\eta I-\gamma I \\
A^{\prime}=(1-f) \nu L-\eta A
\end{array}\right.
$$

with initial data

$$
S\left(t_{0}\right)=S_{0}, L\left(t_{0}\right)=L_{0}, I\left(t_{0}\right)=I_{0}, A\left(t_{0}\right)=A_{0} .
$$

Let us denote $\tau$ the transmission rate at the early stage of the epidemics. It depends on infectiousness of the disease times the number of contact by time unit at time $t_{0}$. At time $t>t_{0}$, susceptible individuals may be contaminated by latent infectious $L(t)$, asymptomatic infectious $A(t)$ or infectious severe symptomatic individuals $I(t)$. On the contrary to Liu et al. [11] we assume that these reported individuals are still active in the transmission of the disease, because many cases of health care workers have been contaminated while they were taking care of infectious severe symptomatic cases. However, we would assume that their transmission rate $\tau \varepsilon_{2}$ is very small compared to the one of latent or asymptomatic individuals by assuming $0<\varepsilon_{2} \ll 1$, to take into account that they are isolated as soon as their symptoms are detected. For latent or asymptomatic individuals, the transmission rate is $\tau \varepsilon_{1}$, where $\varepsilon_{1}$ is by definition equal to 1 before public health measures had been taken. Parameter $\varepsilon_{1} \in[0,1]$ is time dependent in order to take into account the impact of physical distancing. It is a central parameter in our modeling: $\varepsilon_{1}(t)$ represents the number of contacts by time units at time $t$ over the number of contacts people had before the epidemic outbreak. Parameter $\varepsilon_{1}$ will be called the intensity of the contact. This parameter depends on health policy, but also on a behavioral component (protective measures applied by individuals). We consider this parameter as a control parameter in order to study the spread of the epidemic for different values of $\varepsilon_{1}$. 
Current modeling is based on Liu et al. [10, 11] who modeled the spread of the epidemic in both Wuhan and in China, and on Magal and Webb [12] who calibrated the epidemic with early data, on several countries including France. However, there are three main differences in our modeling and theirs. Firstly, they do not consider contamination by reported symptomatic cases. It appears that in France, many cases of contamination occur in hospitals (on March 22nd, it was announced that the first hospital doctor had died as a result of his infection with the virus). Secondly, they do not consider over-mortality caused by the virus. Finally, Liu et al. [10] chose an exponential function to model the reduction of the number of contacts during lockdown. However, as lockdown lasted a long time, exponential function would lead to a too low impact on the last period of the lockdown. Thus we would prefer the original function used by Chowell et al. ([6]).

We have used the calibration defined in Magal and Webb [12] to describe the epidemic before the implementation of lockdown measures. The impact of lockdown has then been calibrated. The objective of our paper is to focus on the impact of strategies for lifting the lockdown.

To describe the evolution of the epidemics, we consider parameters given in Table 1 .

\section{RESULTS}

Let us first consider the reproduction number on the epidemics, based on the dynamical system (2.1). Results of Van den Driessche and Watmough [14] are applied. The linearized dynamics of the infective classes (that is latent $L$, symptomatic $I$ and asymptomatic $A$ ) of System (2.1) around the disease free equilibrium (DFE) given by $\left(S^{*}, 0,0,0\right)$ with $S^{*} \approx S_{0}$, is considered. This linearized dynamics is given as follows.

$$
\left\{\begin{array}{l}
L^{\prime}=\tau S_{0}\left(\varepsilon_{1}(t)(L+A)+\varepsilon_{2} I\right)-\nu L \\
I^{\prime}=f \nu L-\eta I-\gamma I \\
A^{\prime}=(1-f) \nu L-\eta A
\end{array}\right.
$$

The reproduction number is the expected number of cases generated by one infected individual in a population where all individuals are susceptible to infection. Since the model includes parameter $\varepsilon_{1}$, that is influenced by public policy, we would call the reproduction number the control reproduction number, and denote it $\mathcal{R}_{C}$. Formally, the reproduction number is the spectral radius of $F V^{-1}$, where matrix $V$ and $F$ are defined as follows.

$$
V=\left[\begin{array}{ccc}
\nu & 0 & 0 \\
-\nu_{1} & \eta+\gamma & 0 \\
-\nu_{2} & 0 & \eta
\end{array}\right] \text { and } F=\tau S_{0}\left[\begin{array}{ccc}
\varepsilon_{1} & \varepsilon_{2} & \varepsilon_{1} \\
0 & 0 & 0 \\
0 & 0 & 0
\end{array}\right]
$$

Matrix $F-V$ is the Jacobian matrix of System (3.1). $F$ is linked with the terms involved in the transmission of the disease, $V$ in the terms linked to the change of compartment for other reasons that disease transmit.

Thus $\mathcal{R}_{C}$ is explicitly computed as

$$
\mathcal{R}_{C}=\frac{\tau S_{0}}{\nu(\gamma+\eta) \eta}\left[\varepsilon_{1}(\gamma+\eta)\left(\eta+\nu_{2}\right)+\varepsilon_{2} \eta \nu_{1}\right]
$$

Let us define $\varepsilon_{1}^{*}$ as

$$
\varepsilon_{1}^{*}=\frac{\frac{\nu(\gamma+\eta) \eta}{\tau S_{0}}-\varepsilon_{2} \eta \nu_{1}}{(\gamma+\eta)\left(\gamma+\nu_{2}\right)} .
$$

It can be noticed that for $\varepsilon_{1}<\varepsilon_{1}^{*}$, then $\mathcal{R}_{C}<1$, there is no epidemic peak. It can also be seen that $\mathcal{R}_{C}$ is a linear function of the intensity of the contacts $\varepsilon_{1}$. 
To understand the course of the epidemics, we are also interested in characterizing the relationship between the final size of the epidemics, that is the number of susceptible at the end of the epidemics, and the intensity of the contacts. Our approach relies on a method developed by Arino et al. [3] in a general framework applied to various cases. Brauer [4] considers the final size of an epidemic computed with early data, and stresses the importance of taking into account how behavioral components modify the final size of the epidemics.

Let us consider $Y$ a weighted number of infective which is defined by

$$
Y=\frac{1}{S_{0}} L+\frac{\varepsilon_{2}}{\gamma+\eta} \frac{\tau}{\mathcal{R}_{C}} I+\frac{\varepsilon_{1}}{\eta} \frac{\tau}{\mathcal{R}_{C}} A
$$

Then, taking the derivative of $Y$ according to time yields

$$
Y^{\prime}=\tau\left(\varepsilon_{1}(L+A)+\varepsilon_{2} I\right) \frac{S}{S_{0}}-\frac{\tau}{\mathcal{R}_{C}}\left(\varepsilon_{1}(L+A)+\varepsilon_{2} I\right) .
$$

Using equation (3.2) and the first equation of System (2.1) it yields

$$
Y^{\prime}(t)=\left(-\frac{1}{S_{0}}+\frac{1}{\mathcal{R}_{C} S}\right) S^{\prime}(t)
$$

which implies that

$$
\ln \left(\frac{S_{0}}{S}\right)=\mathcal{R}_{C}\left(\frac{S_{0}-S}{S_{0}}+Y_{0}-Y\right)
$$

From the dynamics of susceptible individuals $S$ in System (2.1), it is straightforward that $S$ is non negative strictly decreasing function of $t$. Then $\lim _{t \rightarrow \infty} S(t)$ exists. We would denote $S_{\infty}=\lim _{t \rightarrow \infty} S(t) . S_{\infty}$ is called the final size of the epidemics and measures the number of individuals that have not been contaminated during the epidemics.

If $\mathcal{R}_{C}>1$, then, according to equation (3.3), the weighted number of infective cases first increases up to a maximum value, achieved for $S_{\max }=\frac{S_{0}}{\mathcal{R}_{C}}$. We would denote $t_{\max }$ the time such that $S\left(t_{\max }\right)=S_{\max }$. Thus $S(t)<\frac{S_{0}}{\mathcal{R}_{C}}$ for all $t>t_{\text {max }}$. If $\mathcal{R}_{C}<1$, we obtain that $S(t)<S_{0}<\frac{S_{0}}{\mathcal{R}_{C}}$ for all $t>t_{0}$.

Moreover, it can be proved (see appendix) that $Y_{\infty}=\lim _{t \rightarrow \infty} Y(t)$ exists and satisfies $Y_{\infty}=0$.

The relationship between the final size of the epidemics, initial conditions and parameters is given as follows.

$$
\ln \left(\frac{S_{0}}{S_{\infty}}\right)=\mathcal{R}_{C}\left(\frac{S_{0}-S_{\infty}}{S_{0}}+Y_{0}\right)
$$

Using the definition of $Y_{0}$ gives

$$
\ln \left(\frac{S_{0}}{S_{\infty}}\right)=\mathcal{R}_{C}\left(\frac{S_{0}-S_{\infty}}{S_{0}}+\frac{L_{0}}{S_{0}}\right)+\tau \frac{\varepsilon_{1}}{\eta} A_{0}+\tau \frac{\varepsilon_{2}}{\eta+\gamma} I_{0}
$$

The final size of the epidemics, defined by implicit equation (3.4), strongly depends on the parameters of the model, and in particular on $\varepsilon_{1}$. Formally, it yields

$$
\frac{\mathrm{d} S_{\infty}}{\mathrm{d} \varepsilon_{1}}=\frac{\tau}{\eta \nu} \frac{\left(S_{0}-S_{\infty}+L_{0}\right)\left(\eta+\nu_{2}\right)+\nu A_{0}}{\frac{\mathcal{R}_{C}}{S_{0}}-\frac{1}{S_{\infty}}} .
$$




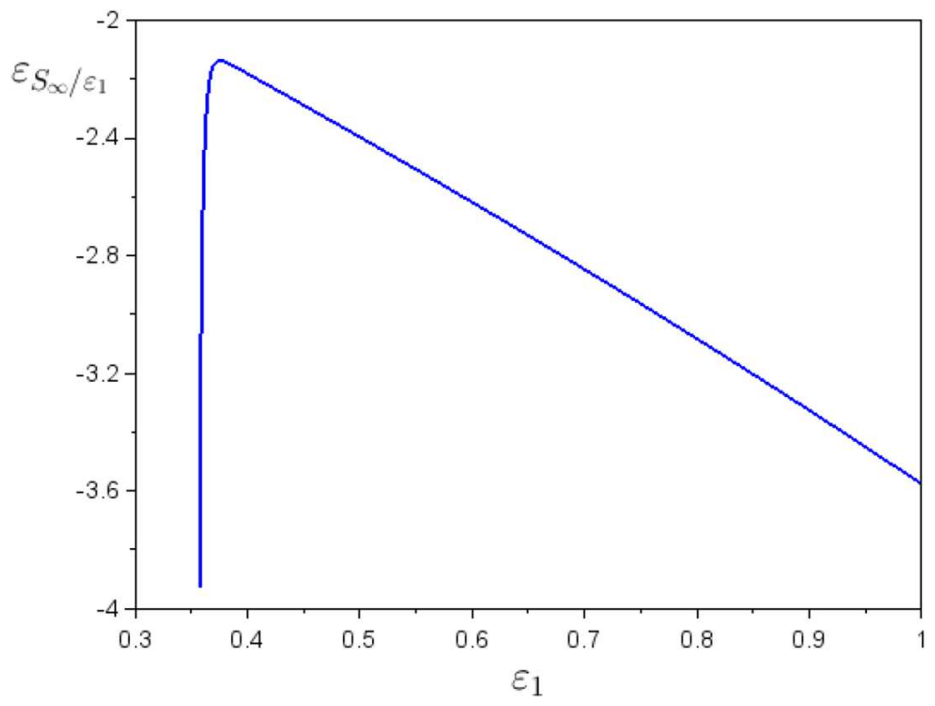

FIgURE 1 . Elasticity $\varepsilon_{S_{\infty} / \varepsilon_{1}}$ of final size $S_{\infty}$ according to contact intensity $\varepsilon_{1}$ for various values of contact intensity $\varepsilon_{1}$.

As we have seen that $S_{\infty}<\frac{S_{0}}{\mathcal{R}_{C}}$, the more the intensity of the contact, the less the final size of the epidemics. To quality this impact, we would prefer to compute the elasticity of $S_{\infty}$ according to $\varepsilon_{1}$ defined as:

$$
\varepsilon_{S_{\infty} / \varepsilon_{1}}=\frac{\frac{\mathrm{d} S_{\infty}}{S_{\infty}}}{\frac{\mathrm{d} \varepsilon_{1}}{\varepsilon_{1}}} .
$$

It indicates the percentage of reduction of the final size of the epidemics, when the intensity of the contact increases by $1 \%$.

Figure 1 gives quantitative value of elasticity $\varepsilon_{S_{\infty} / \varepsilon_{1}}$ using calibration for COVID-19 specified in Table 1. Not surprisingly, in Figure 1, it can be seen that $\varepsilon_{S_{\infty} / \varepsilon_{1}}<0$. It means the greater the intensity of the contact among individuals, the smaller the final size of the epidemics. However, Figure 1 also shows that elasticity $\varepsilon_{S_{\infty} / \varepsilon_{1}}$ is not a monotonous function of $\varepsilon_{1}$. Reducing the intensity of contact of one percent compare to business-as-usual situation $\varepsilon_{1}=1$ enables to increase the final size of the epidemics of $3.57 \%$. When $\varepsilon_{1}$ is close to 0.4 , reducing the intensity of the contact of $1 \%$ increases the final size of the epidemics of $2.18 \%$. When $\varepsilon_{1}$ is close to $\varepsilon_{1}^{*}$, the marginal effort of reducing contact intensity has a huge impact on the size of the epidemics.

Let us consider the number of individuals that have been contaminated during the epidemics, denoted $C_{\infty}$. They correspond to those who have recovered or who are dead from the disease. According to System (2.1), $C_{\infty}$ is obtained as $C_{\infty}=S_{0}-S_{\infty}$. Then

$$
\frac{\mathrm{d} C_{\infty}}{\mathrm{d} \varepsilon_{1}}=-\frac{\mathrm{d} S_{\infty}}{\mathrm{d} \varepsilon_{1}}
$$

and elasticity $\varepsilon_{C_{\infty} / \varepsilon_{1}}$, which is defined as

$$
\varepsilon_{C_{\infty} / \varepsilon_{1}}=\frac{\frac{\mathrm{d} C_{\infty}}{C_{\infty}}}{\frac{\mathrm{d} \varepsilon_{1}}{\varepsilon_{1}}}
$$


TABLE 1. Table of parameters.

\begin{tabular}{llc}
\hline Symbol & Interpretation & Value \\
\hline$t_{0}$ & Time when the epidemic started & February 3rd [12] \\
$t_{1}$ & Time when the lockdown started & March 17th [15] \\
$t_{2}$ & Time when the lockdown ends & May 11th \\
$S_{0}$ & Number of susceptible at time $t_{0}$ & $66.99 \times 10^{6}$ \\
$L_{0}$ & Number of latent infectious at time $t_{0}$ & $3.675[12]$ \\
$A_{0}$ & Number of asymptomatic & $0.892[12]$ \\
& infectious cases at time $t_{0}$ & 1 \\
$I_{0}$ & Number of symptomatic infectious & \\
& infectious cases at time $t_{0}$ & $4.23 \times 10^{-9}[12]$ \\
$\tau$ & Transmission rate at early stage & fitted \\
$\gamma$ & Death due to the disease & 7 \\
$\nu^{-1}$ & Average duration of the asymptomatic infectious period & 0.4 \\
$f$ & Fraction of asymptomatic infectious who \\
$\nu_{1}=f \nu$ & become reported symptomatic infectious & \\
$\nu_{2}=(1-f) \nu$ & Rate at which asymptomatic infectious & \\
$\eta^{-1}$ & become reported symptomatic infectious & \\
$\varepsilon_{1}(t)$ & Rate at which asymptomatic infectious & \\
$\varepsilon_{2}$ & Aveome unreported symptomatic infectious & 7 \\
& Number of contacts by time unit at time $t$ & control \\
\hline
\end{tabular}

satisfies

$$
\varepsilon_{C_{\infty} / \varepsilon_{1}}=-\frac{\tau}{\eta \nu} \frac{\left(C_{\infty}+L_{0}\right)\left(\eta+\nu_{2}\right)+\nu A_{0}}{\frac{\mathcal{R}_{C}}{S_{0}}-\frac{1}{S_{\infty}}} \frac{\varepsilon_{1}}{C_{\infty}} .
$$

Non surprisingly, it can be seen in Figure 2 that $\varepsilon_{C_{\infty} / \varepsilon_{1}}>0$. It means that the total number of individuals who have recovered or who are dead from the epidemics is increasing is $\varepsilon_{1}$; the more the intensity of the contact the more infected individuals. However, elasticity $\varepsilon_{C_{\infty} / \varepsilon_{1}}$ is a decreasing function of $\varepsilon_{1}$. If the intensity of the contact is close to its level before the epidemic outbreak (that is $\epsilon_{1}$ close to 1 ), then a reduction of $1 \%$ of the intensity of the contact yields to a reduction of $0.18 \%$ of $C_{\infty}$. If the intensity of the contact is half the one it was before the epidemic outbreak, a reduction of $1 \%$ of the intensity of the contact yields to a reduction of $1.36 \%$ of $C_{\infty}$.

\section{NumERICAL EXPERIMENTS}

Data from the French Public Health Agency from February 25th to the 1st of June have been used.

Calibration of the epidemics in the early phase of the epidemics is obtained using the results of Liu et al. [10] and are given in Table 1 . The starting time of our simulations is taken as $t_{0}$ being February the 3rd.

During lockdown.

The effect of lockdown is to reduce the number of the contacts by time unit. The impact of this reduction is modeled through parameter $\varepsilon_{1}$, which was set to 1 before public health measures had been taken. The impact of these interventions is not instantaneous, as it was stressed by Chowell et al. [6]. This impact can be modeled using exponential form, which is the case used by Althaus [1] for 2014 Ebola virus outbreak or by Liu et al. [11] concerning the early stage of COVID-19 epidemics. However, as the lockdown period lasted a long time, 


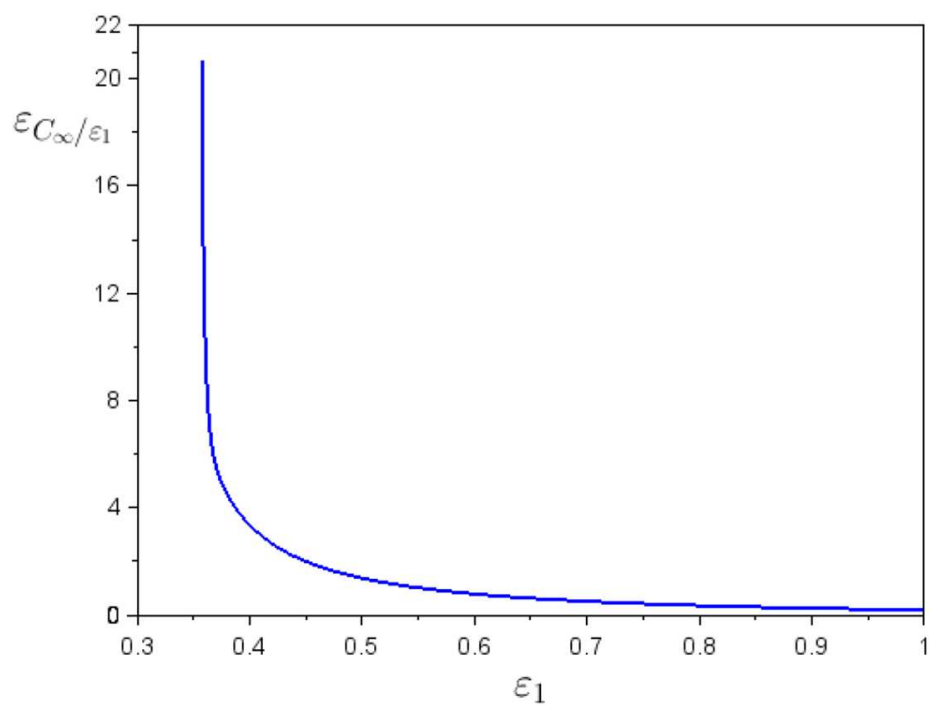

FigURE 2. Elasticity $\varepsilon_{C_{\infty} / \varepsilon_{1}}$ of number of individuals that have been contaminated at the end of the epidemics $C_{\infty}$ according to contact intensity $\varepsilon_{1}$ for various values of contact intensity $\varepsilon_{1}$.

we would prefer to use a form similar to Chowell et al. [6], for which $\varepsilon_{1}(t)$ decreases gradually from 1 to $\varepsilon_{1}^{1}$, as given by equation (4.1), as follows.

$$
\varepsilon_{1}(t)=\left\{\begin{array}{l}
1 \text { for } t<t_{1}, \\
\varepsilon_{1}^{1}+\left(1-\varepsilon_{1}^{1}\right) e^{-\mu\left(t-t_{1}\right)}, \text { for } t>t_{1}
\end{array}\right.
$$

Parameters $\varepsilon_{1}^{1}, \mu$ and $\gamma$ have been calibrated using least-squares fitting. The following results have been obtained: $\varepsilon_{1}^{1}=0.1828483, \mu=0.1424125$ and $\gamma=0.0203774$. Thus contacts have reduced to $5-6$ fold in France (compared to 7-8 fold in Wuhan and Shangai [16]). Figure 3 presents our simulations compared to data of the Public Health Agency from February 25th to the 1st of June on daily number of symptomatic cases $(f \nu L(t))$ (Fig. 3a), on the daily number of deaths (Fig. 3b).

The comparison of our simulations to data of the Public Health Agency as far as cumulative number of reported cases and cumulative number of deaths is concerned is given in Figure 4.

Lockdown lifting.

At time $t_{2}$ taken as May 11th, the lockdown ended in France, but a number of restrictive measures still apply: movement restriction and schools, restaurants and bars remained closed. Different scenarios for the end of the lockdown which are compatible with data available on June 1st are now presented. On the contrary to Ferguson et al. [8], we only consider one specific time for the start and end of lockdown. We assume that the intensity of the contact $\varepsilon_{1}$ can be modeled by equation (4.2).

$$
\varepsilon_{1}(t)=\left\{\begin{array}{l}
1 \text { for } t<t_{1} \\
\varepsilon_{1}^{1}+\left(1-\varepsilon_{1}^{1}\right) e^{-\mu\left(t-t_{1}\right)} \text { for } t_{1}<t<t_{2}, \\
\frac{e^{c\left(t-t_{2}\right)}}{\frac{1}{\varepsilon_{1}^{1}+\left(1-\varepsilon_{1}^{1}\right) e^{-\mu\left(t_{2}-t_{1}\right)}}-G+G e^{c\left(t-t_{2}\right)}} \text { for } t>t_{2} .
\end{array}\right.
$$

Function $\varepsilon_{1}(t)$ is continuous, and for $t>t_{2}, \varepsilon_{1}(t)$ satisfies the following differential equation.

$$
\varepsilon_{1}^{\prime}(t)=c \varepsilon_{1}(t)\left(1-G \varepsilon_{1}(t)\right)
$$




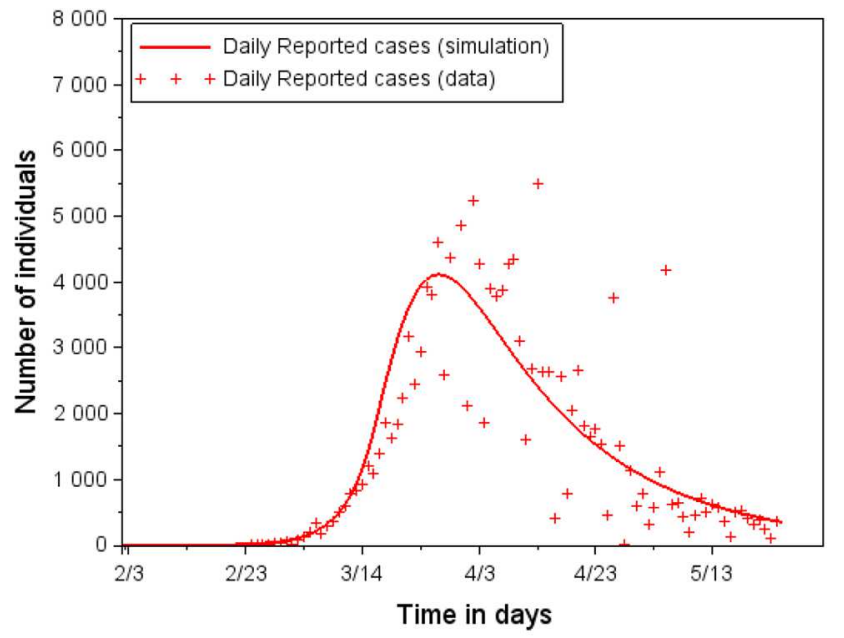

(a)

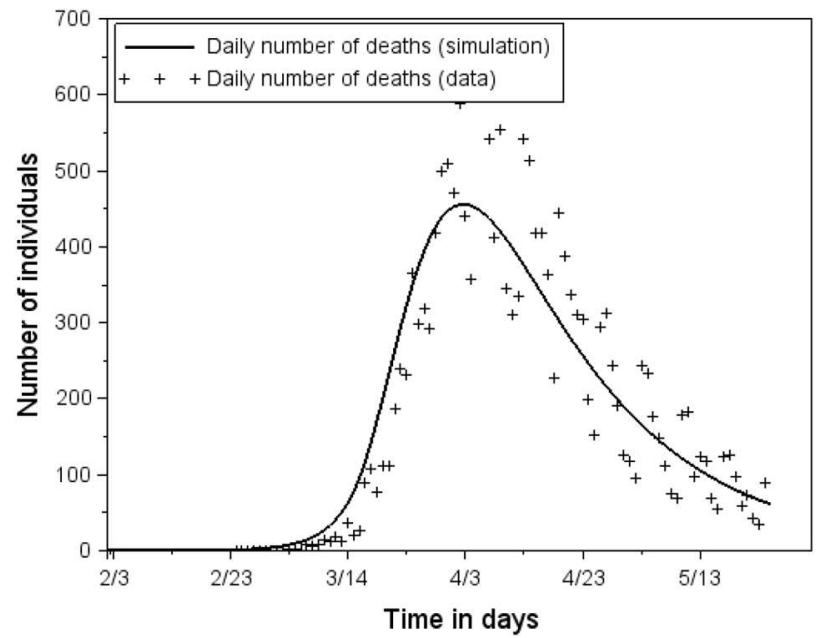

(b)

Figure 3. Daily number of reported symptomatic cases (a) and daily number of COVID-19 deaths (b) for France.

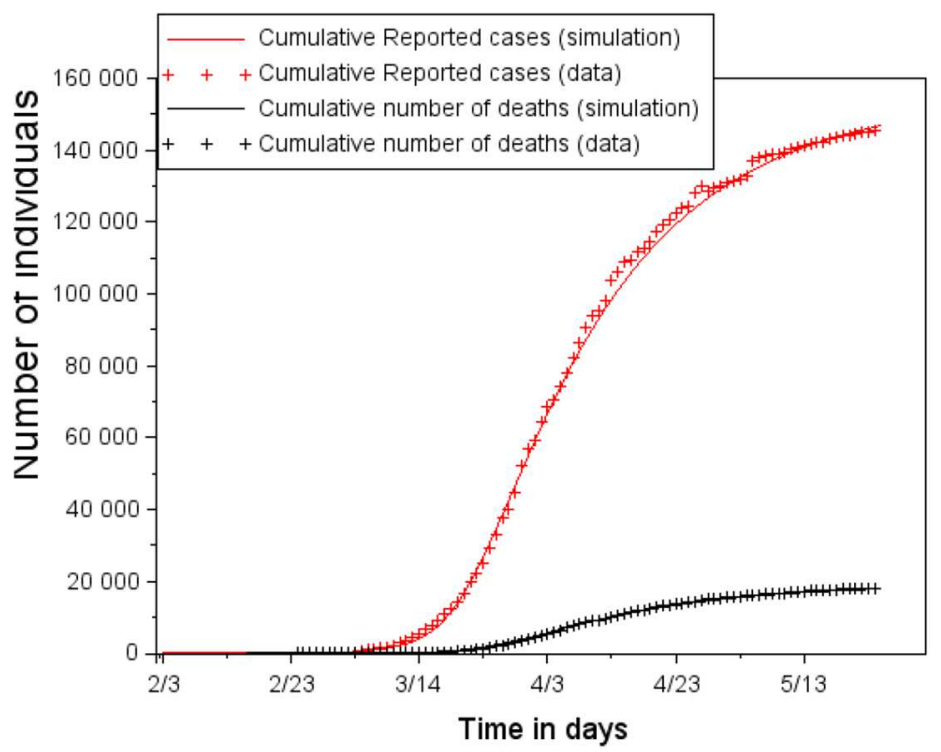

Figure 4. Cumulative number of reported symptomatic cases and deaths for France.

Thus, after the lift of lockdown, intensity of contact is modeled by a logistic function which reflects that physical distancing habits acquired during lockdown period would be progressively lost as time goes on. Modeling health habit with logistic function has been used by Fournier et al. [9]. In our modeling $c$ is the speed of the increase contact intensity and $G^{-1}$ is the long run contact intensity.

We would first consider three scenarios modeling contact intensity after the lift of lockdown. These scenarios would depend on parameters $c$ and $G$. A first scenario is a fast loss of physical distancing habit acquired during 


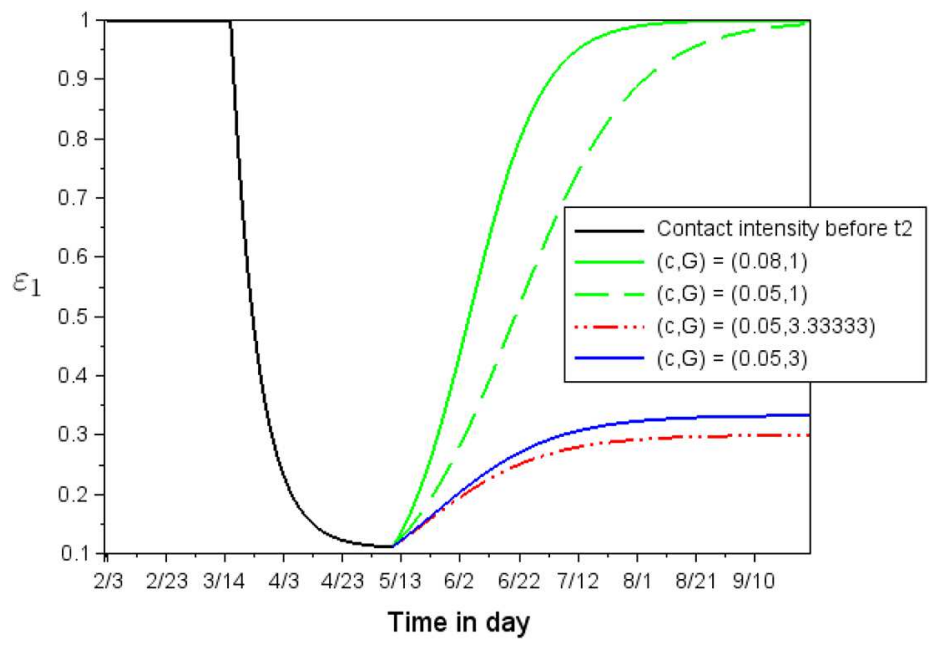

FIGURE 5. Contact intensity function $\varepsilon_{1}$ according the various scenarios.

lockdown period and convergence to contact intensity as it was before lockdown. However, to take into account the three phases of the lift of lockdown in France, immediate convergence to contact intensity as it was before the epidemic outbreak is excluded. Thus, this first scenario will be obtained by considering $(c, G)=(0.08,1)$. The second scenario is a slow loss of physical distancing habits and in the long run, contact intensity is the same as the one before the epidemic outbreak. This second scenario corresponds to $(c, G)=(0.05,1)$. In these two scenarios, we would call business-as-usual the situation of contact intensity in the long run. A third scenario is also considered. It corresponds to a slow loss of social distancing habits with individuals continuing to respect advices to stop the virus spread and physical distancing even in the long run. In this scenario, we would assume that contact intensity is a third of what it was before the epidemic outbreak by assuming $(c, G)=(0.05,3)$.

The value of contact intensity $\varepsilon_{1}$ for these three scenarios are plotted in Figure 5 .

Simulations obtained from System (2.1) using functional form of contact intensity given by equation (4.2) are now considered. Parameters are given in Table 1 , together with $\varepsilon_{1}^{1}=0.1828483, \mu=0.1424125$ and $\gamma=$ 0.0203774 (as explained previously). Various choices of values for $(c, G)$ will generate various scenarios. We will first consider the three scenarios described previously corresponding to $(c, G)=(0.08,1)$ (scenario 1 ), $(c, G)=(0.05,1)$ (scenario 2) and $(c, G)=(0.05,3)$ (scenario 3). A fourth scenario corresponding to $(c, G)=$ $(0.05,3.33333)$ will be also considered.

The trajectories of daily symptomatic reported cases and daily deaths are presented in Figure 6 based on the various scenarios. It can be seen that with the business-as-usual scenario (with either a fast or slow end to the lockdown) a major peak of the epidemic appears in summer (Fig. 6a and b). The only differences between a fast and slow end to lockdown are that for the fast end scenario, the timing of the peak would be reached sooner (end of July with in Fig. 6a and end of August in Fig. 6b) and its magnitude would be greater with fast end to lockdown. Even if individuals reduce their contact habits to a third of what they were before the epidemic outbreak, this is still not enough to prevent a major epidemic outbreak that would happen by the end of the year.

In Figure $6 \mathrm{~d}$ a fourth scenario is considered. It corresponds to a slow loss of physical distancing habits, with contact habits in the long run being reduced to $70 \%$ of what they were, and it is obtained by considering $(c, G)=\left(0.05,0.3^{-1}\right)$. With this scenario, there is no re-emergence of the epidemic. Beyond this threshold, there is re-emergence of the epidemic. This threshold corresponds 1.8 times the contact intensity achieved by May 11th. 


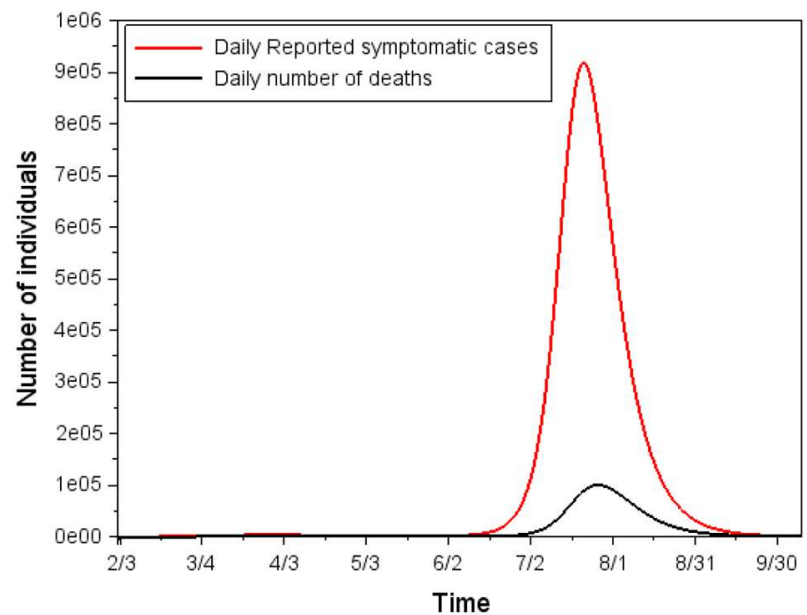

(a)

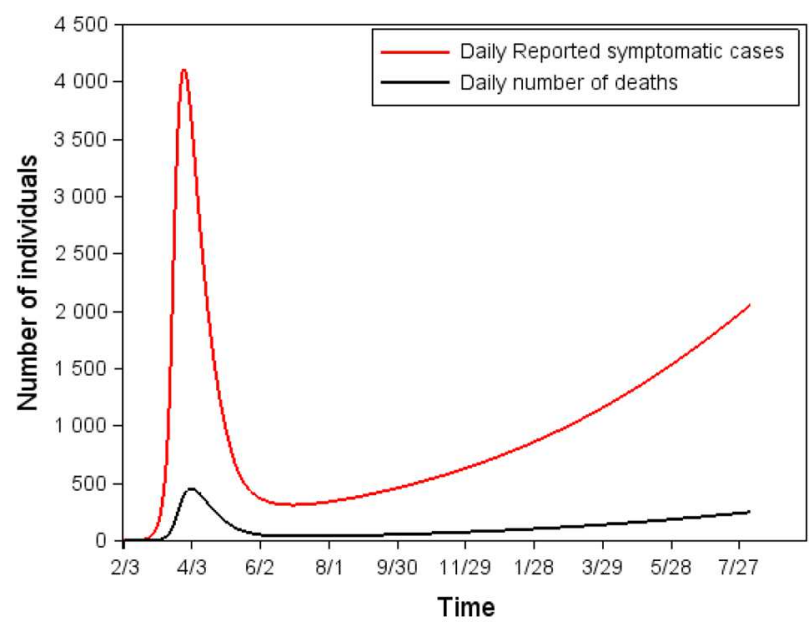

(c)

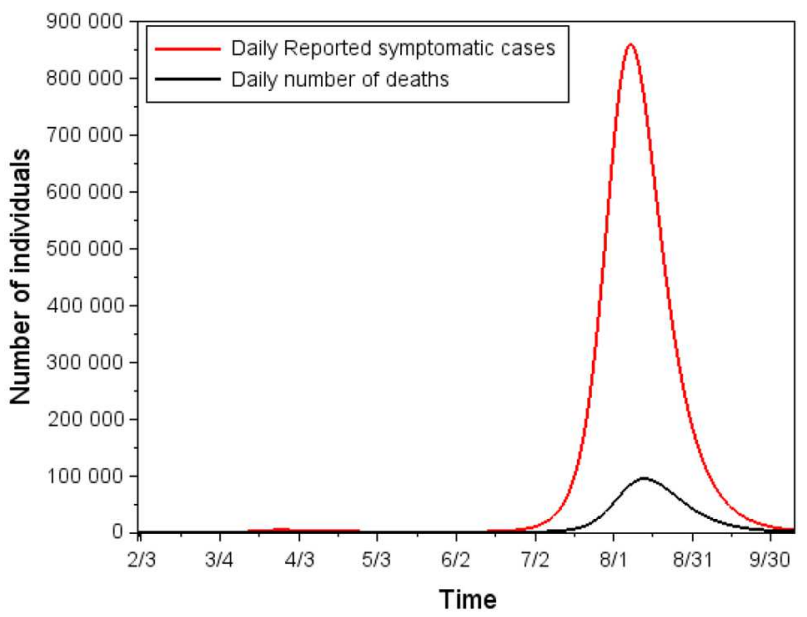

(b)

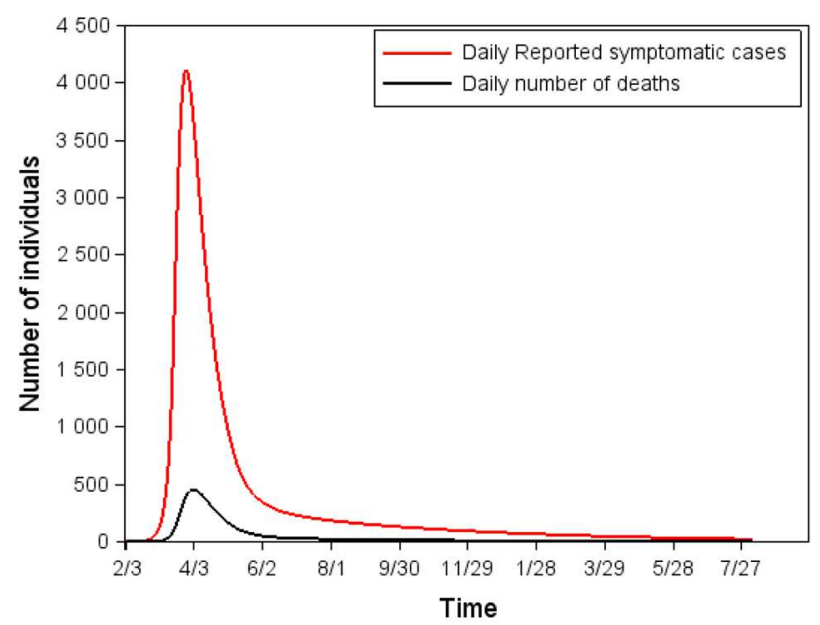

(d)

FiguRE 6. Daily number of reported cases and daily number of deaths according to the various scenarios. $(c, G)=(0.08,1)$ (scenario 1) panel (a). $(c, G)=(0.05,1)$ (scenario 2) panel (b). $(c, G)=(0.05,3)$ (scenario 3) panel $(\mathrm{c}) \cdot(c, G)=\left(0.05,0.3^{-1}\right)$ (scenario 4) panel (d).

\section{Discussion}

France was the 7th country to be affected by the COVID-19 virus and delayed the strict lockdown application. By that time, the number of symptomatic reported cases was already large (6633 reported cases on March the $17 \mathrm{th}$ ) and lockdown has not prevented deaths and contamination of a large part of the population. However, it has drastically slowed down the spread of the virus. The economic loss is huge (production has decreased by one third) and lockdown can only be a temporary measure. This is the reason for studying the end-of-lockdown measures in this paper. We have seen that only scenarios with drastic reduction of physical distancing compared to the one that prevailed before the epidemic outbreak prevent from the re-emergence of the disease. 


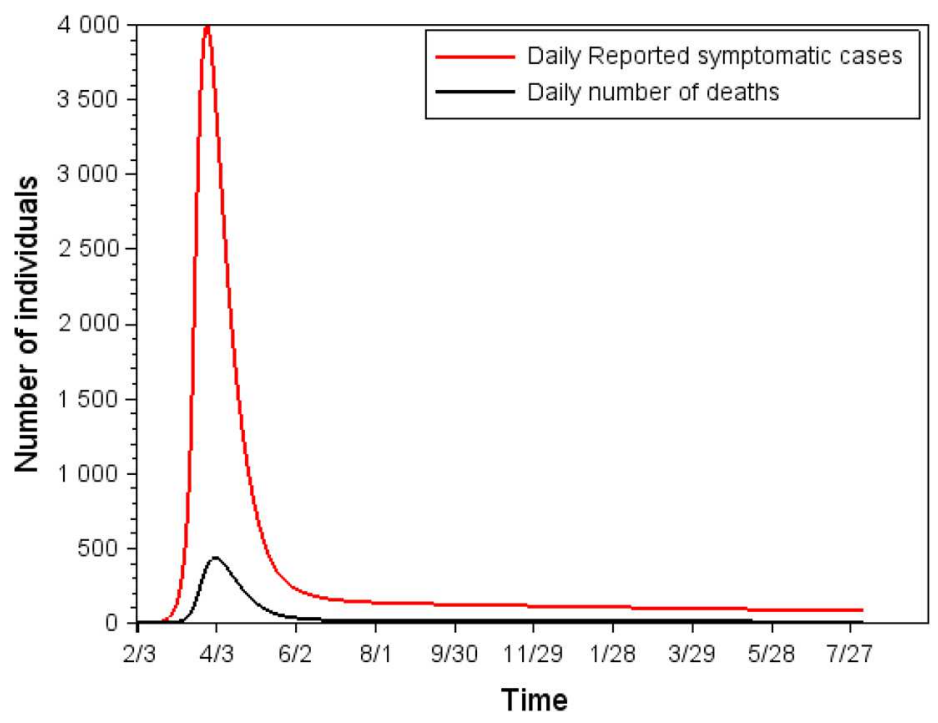

FiguRE 7. Daily number of reported cases and daily number of deaths with $(c, G)=(0.05,3)$ (scenario 3 ) and $f=0.6$ (instead of $f=0.4$ in the previous figures).

To avoid the re-emergence of the epidemics after the end of lockdown, public policies still need to be applied, as for example a more intensive screening process. Figure 7 shows scenario $3((c, G)=(0.05,3))$ with the addition of a more intense screening process. It can be seen that this would avoid the re-emergence of the disease.

Another possible health policy measure might be to lift the lockdown for only one part of the population. We would consider a realistic scenario with slow loss of intensity of contact habits acquired during the lockdown and reduction of one half of the intensity of contact in the long run from what it was before the epidemic outbreak. This corresponds to $(c, G)=(0.05,2)$. Figure 8 presents the evolution of the epidemics under various scenarios which differ the one from another by the number of individuals concerned by the lift of lockdown. It is assumed in Figure 8a that the end of lockdown applies to all the population. The peak of the epidemics appears in November 2020. In Figure 8b we consider the case in which $30 \%$ of the population is contained and its contact intensity is similar to its value at the end of the lockdown period. The peak of the epidemics appears in February 2021. In Figure 8c that it is assumed that $60 \%$ of the population is maintained under a lockdown regime. It can be seen that there is no re-emergence of the disease.

\section{Supplementary material}

\subsection{Proof of $Y_{\infty}=0$}

We have proved that there exists $t_{m} \geq 0$ such that $Y(t)$ is a decreasing function of $t$, for $t>t_{m}$. Moreover, from equation (2.1), $L(t), A(t)$ and $I(t)$ are non negative functions, which implies that $Y(t) \geq 0$.

Let us call $D(t)$ the number of deaths. From equation (2.1),

$$
S^{\prime}+L^{\prime}+I^{\prime}+A^{\prime}+D^{\prime}=-\eta(I+A) .
$$

Thus $S+L+I+A+D$ is a decreasing function and $S(t)+L(t)+I(t)+A(t)+D(t) \leq N(0)$, where $N(0)=$ $S(0)+L(0)+A(0)$. Moreover

$$
\eta \int_{0}^{t}(I+A) \leq N(0)
$$




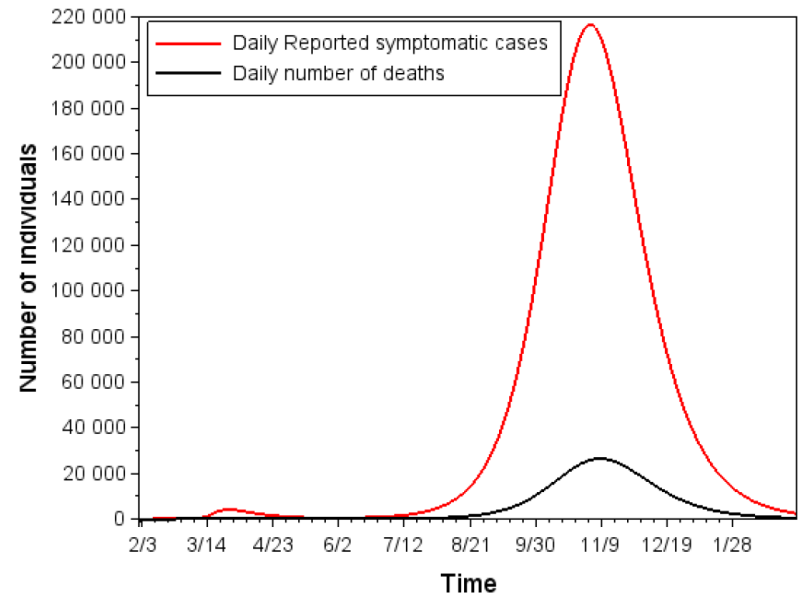

(a)

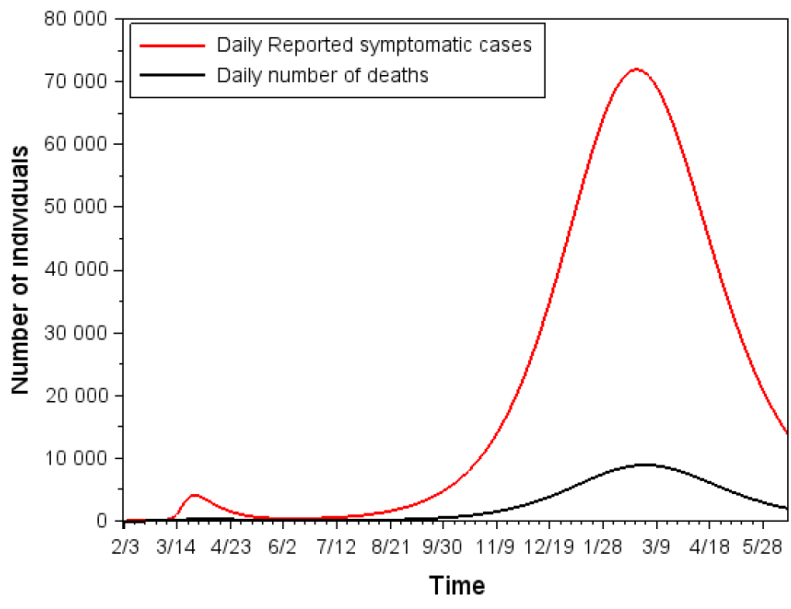

(b)

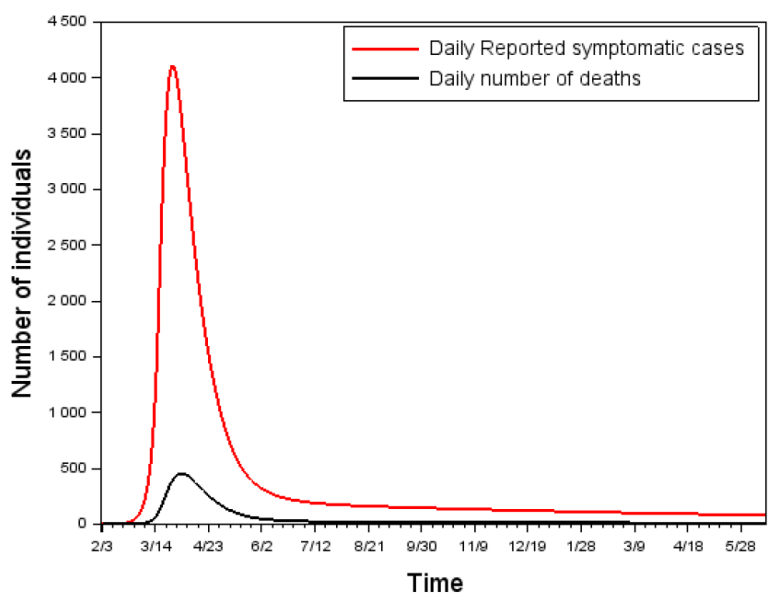

(c)

Figure 8. Daily number of reported cases and daily number of deaths with $(c, G)=(0.05,2)$ under various scenarios of partial lockdown. Lift of lockdown for all the population (a). Lift of lockdown for $70 \%$ of the population (b). Lift of lockdown for $40 \%$ of the population (c).

thus $\int_{0}^{t}(I+A)<\infty$, which implies that $\lim _{t \rightarrow \infty} I(t)=\lim _{t \rightarrow \infty} A(t)=0$.

As $A^{\prime}(t)=(1-f) \nu L(t)-\eta A(t)$, then

$$
A(t)=A(0) e^{-\eta t}+(1-f) \nu \int_{0}^{t} L(s) e^{-\eta(t-s)} \mathrm{d} s
$$

which implies that $\lim _{t \rightarrow \infty} L(t)=0$.

Thus $Y(t)=0$.

\section{REFERENCES}

[1] C.L. Althaus, Estimating the reproduction number of Ebola virus (EBOV) during the 2014 outbreak in West Africa. PLoS Curr. 6 (2014). 
[2] J. Arino, F. Brauer, P. van den Driessche, J. Watmough and J. Wu, Simple models for containment of a pandemic. J. R. Soc. Interface 3 (2006) 453-457.

[3] J. Arino, F. Brauer, P. Van Den Driessche, J. Watmough and J. Wu, A final size relation for epidemic models. Math. Biosci. Eng. 4 (2007) 159.

[4] F. Brauer, Early estimates of epidemic final sizes. J. Biol. Dyn. 13 (2019) 23-30.

[5] F. Brauer, The final size of a serious epidemic. Bull. Math. Biol. 81 (2019) 869-877.

[6] G. Chowell, N.W. Hengartner, C. Castillo-Chavez, P.W. Fenimore, J.M. Hyman, The basic reproductive number of Ebola and the effects of public health measures: the cases of Congo and Uganda. J. Theor. Biol. 229 (2004) 119-126.

[7] A.B. Gumel, S. Ruan, T. Day, J. Watmough, F. Brauer, P. Van den Driessche and J. Wu, Modelling strategies for controlling SARS outbreaks. Proc. R. Soc. Lond. Ser. B 271 (2004) 2223-2232.

[8] N. Ferguson, D. Laydon, G. Nedjati Gilani, N. Imai, K. Ainslie, M. Baguelin and A. Dighe, Report 9: Impact of non-pharmaceutical interventions (NPIs) to reduce COVID19 mortality and healthcare demand (2020).

[9] M. Fournier, F. d'Arripe-Longueville, C. Rovere, C.S. Easthope, L. Schwabe, J. El Methni and R. Radel, Effects of circadian cortisol on the development of a health habit. Health Psychol. 36 (2017) 1059.

[10] Z. Liu, P. Magal, O. Seydi and G. Webb, Understanding unreported cases in the 2019-nCov epidemic outbreak in Wuhan, China, and the importance of major public health interventions. MPDI Biol. 9 (2020) 50.

[11] Z. Liu, P. Magal, O. Seydi and G. Webb, Predicting the cumulative number of cases for the COVID-19 epidemic in China from early data. arXiv preprint arXiv:2002.12298 (2020).

[12] P. Magal and G. Webb, Predicting the number of reported and unreported cases for the COVID-19 epidemic in South Korea, Italy, France and Germany. Italy, France and Germany, March 19, 2020.

[13] A. Mignon Colombet and D. Floreancig, L'infraction de "mise en danger d'autrui" est à manier avec précaution , Tribune, Le Monde, 25 mars 2020.

[14] P. Van den Driessche and J. Watmough, Reproduction numbers and sub-threshold endemic equilibria for compartmental models of disease transmission. Math. Biosci. 180 (2002) 29-48.

[15] https://en.wikipedia.org/wiki/2020_coronavirus_outbreak_in_France

[16] J. Zhang, M. Litvinova, Y. Liang, Y Wang, W. Wang, S. Zhao and M. Ajelli, Changes in contact patterns shape the dynamics of the COVID-19 outbreak in China. Science 368 (2020) 1481-1486. 